\title{
Amyloidosis: Rare or Underdiagnosed Disease?
}

\author{
Mariano Napoli Llobera ${ }^{1 \oplus}$ and Atilio Costa Vitali ${ }^{\circledR}$ \\ Research fellowship, Division of Cardiology, Health Sciences North Hospital, Sudbury, ${ }^{1}$ Ontario - Canada \\ Medical Director of Heart Failure Disease Management Program, Division of Cardiology, Health Sciences North Hospital, ${ }^{2}$ Sudbury, \\ Ontario - Canada \\ On behalf of the Council of Myocardiopathy of the Inter-American Society of Cardiology
}

Amyloidosis is the general term for a clinical condition caused when one of more than 30 different precursor proteins with unstable tertiary structure misfolds and aggregates as insoluble amyloid fibrils which deposit in the extracellular space of organs and soft tissue. Classification of amyloidosis is based upon the type of precursor protein. ${ }^{1,2}$

Specifically, cardiac amyloidosis (CA) is a type of restrictive cardiomyopathy, in which the infiltration of amyloid fibrils into myocardial tissue results in progressive ventricular stiffness, wall thickening, and diastolic dysfunction due to restrictive physiology, which typically manifests as heart failure with preserved ejection fraction (HFpEF). When the disease is advanced, systolic dysfunction might also be seen. ${ }^{3,4}$

CA can clinically simulate other cardiovascular diseases; therefore, it is believed to be underdiagnosed, and the true prevalence and incidence are uncertain. ${ }^{2}$

Among the many types of amyloidosis, nearly all cases of clinical CA (> 95\%) are caused by transthyretin amyloidosis (ATTR) or light chain amyloidosis (AL).

\section{Transthyretin amyloidosis}

Transthyretin amyloidosis (ATTR) is caused by the deposition of transthyretin (TTR), a protein synthesized by the liver, which has a stable tetrameric structure and whose function is the transport of thyroid hormones and retinol. TTR can dissociate into monomers and oligomers and then be deposited as amyloid fibrils, in a natural (wtATTR or "wild type", previously known as senile systemic amyloidosis) or genetic manner (hATTR or "hereditary"). ${ }^{4}$

The number of patients diagnosed with ATTR has increased over the years, and it has come to be considered that it may be more prevalent than AL.

According to different studies, ATTR deposits have been identified in $25 \%$ of autopsies in patients older than 80 years; using non-invasive imaging methods, it has been estimated that ATTR represents $13 \%$ of patients with HFpEF and $16 \%$ of those who undergo transcutaneous

\section{Keywords}

Amyloidosis; Heart Failure; Restrictive Cardiomyopathy.

\section{Mailing Address: Mariano Napoli Llobera •}

41 Ramsey Lake Road, Sudbury ON P3E 5J1, Canadá

E-mail: mariano.napoli1@gmail.com

Manuscript received September 09, 2021; revised manuscript September 24, 2021; accepted September 24, 2021.

DOI: https://doi.org/10.36660/abchf.20210019 aortic valve replacement for severe low-flow, lowgradient aortic stenosis with preserved ejection fraction (paradoxical). ${ }^{5}$

If the instability of the tetrameric structure of TTR is due to a genetic mutation, a predominantly neurological or cardiac condition will occur. hATTR is considered a rare disease, with a prevalence of less than 1 per 100,000 inhabitants. Currently there are more than 120 mutations that can cause ATTR, and the most frequent mutation worldwide is Val30Met, which is endemic in Portugal, Sweden, Japan, Brazil, and Spain. This mutation can cause a symmetric and ascending sensorimotor polyneuropathy, dysautonomia, and in $43 \%$ of cases cardiac involvement. ${ }^{5}$

\section{Immunoglobulin light chain amyloidosis}

Immunoglobulin light chain amyloidosis (AL) is caused by multisystem deposition of immunoglobulin light chains, as a consequence of plasma cell dyscrasia. The currently reported incidence is 1 in 100,000 people, being more frequent in those over 40 years of age, compromising the liver, lungs, kidneys, spleen, and the autonomic and peripheral nervous systems. Cardiac involvement, which occurs in $50 \%$ to $70 \%$ of cases, is considered a prognostic factor, because light chains have greater cardiotoxicity than TTR, and, consequently, a lower response to heart failure treatment. ${ }^{6}$

It is important to clinically differentiate between ATTR and $A L$, as they have different clinical courses.

In general, ATTR presents slowly progressive development, and it is more frequent in older male adults. $\mathrm{AL}$ generally has a rapidly progressive course, presenting at younger ages with less male predominance. ${ }^{4}$

\section{Diagnosis and management}

AC should be suspected when the patient presents symptoms and signs of HFpEF with unexplained increased left ventricular wall thickness $(>12 \mathrm{~mm}$ ) and 1 or more clinical manifestations. ${ }^{5,7,8}$ (Table 1)

Clinical signs of CA with echocardiography, cardiac magnetic resonance, elevated biomarkers, and grade 2-3 uptake with 99mTc-PYP (pyrophosphate) in scintigraphy, with the exclusion of a monoclonal protein that could cause $\mathrm{AL}$ by the light chain test in serum and urine, confer a positive predictive value of $100 \%$ for the diagnosis of ATTR. ${ }^{8}$ (Table 2 )

In these cases, biopsy is not required, and genetic testing should be performed to distinguish a "hereditary" from "wild" variant. When AL is suspected, biopsy of bone marrow and other tissues (fat pad, salivary, or endomyocardial) is 


\section{Editorial}

\begin{tabular}{|c|c|c|c|}
\hline Extra-cardiac manifestations & AL & hATTR & wtATTR \\
\hline Renal & $\begin{array}{l}\text { Renal insufficiency } \\
\text { Nephrotic syndrome }\end{array}$ & \multicolumn{2}{|c|}{ Milder renal insufficiency (mainly due to heart failure) } \\
\hline Autonomic & \multicolumn{3}{|c|}{$\begin{array}{l}\text { Orthostatic hypotension } \\
\text { Gastroparesis } \\
\text { Sexual dysfunction }\end{array}$} \\
\hline Neurologic & \multicolumn{3}{|c|}{$\begin{array}{l}\text { Peripheral sensorimotor neuropathy (predominant in hATTR) } \\
\text { Carpal tunnel syndrome (bilateral) } \\
\text { Spinal stenosis (predominantly lumbar) }\end{array}$} \\
\hline Musculoskeletal & \multicolumn{3}{|c|}{$\begin{array}{l}\text { Muscle weakness } \\
\text { Arthropathy } \\
\text { Fatigue } \\
\text { Cachexia }\end{array}$} \\
\hline Gastrointestinal & $\begin{array}{l}\text { a, constipation, early satiety, } \\
\text { abdominal bloating }\end{array}$ & \multicolumn{2}{|c|}{ Elevated liver enzymes } \\
\hline Hematologic & \multicolumn{3}{|c|}{ Bleeding and periorbital bruising } \\
\hline Ocular manifestations & \multicolumn{3}{|c|}{ Vitreous opacities } \\
\hline \multicolumn{4}{|c|}{$\begin{array}{l}\text { AL: light chain amyloidosis; hATTR: hereditary transthyretin amyloidosis; wtATTR: wild type transthyretin amyloidosis } \\
\text { Adapted from Fine M, Davis M, Anderson K, Delgado D, et al. Canadian Cardiovascular Society/Canadian Heart Failure Society Joint Position Statement } \\
\text { on the Evaluation and Management of Patients with Cardiac Amyloidosis. CJCA. 2020; 20:322-334 }\end{array}$} \\
\hline Electrocardiography & Echocardiography & Biomarkers & $\begin{array}{l}\text { Cardiac magnetic } \\
\text { resonance }\end{array}$ \\
\hline $\begin{array}{l}\text {-Low voltage QRS (disproportional to LV wall } \\
\text { thickness) } \\
\text {-Poor R-wave progression (pseudoinfarction } \\
\text { pattern) } \\
\text { abnormalities } \\
\text {-Left bundle branch block } \\
\text {-Atrial fibrillation } \\
\text {-AV block }\end{array}$ & $\begin{array}{c}\text {-Unexplained LV hypertrophy } \\
\text {-Biatrial dilatation } \\
\text {-RV free wall hypertrophy } \\
\text {-Speckled appearance of the myocardium } \\
\text {-Diastolic LV dysfunction } \\
\text {-Low flow, low gradient aortic stenosis } \\
\text {-Abnormal GLS with apical sparing } \\
\text { ("cherry on top" pattern } \\
\text {-Apical strain/average of mid and basal } \\
>1.0 \\
\text {-Moderate pulmonary Hypertension } \\
\text {-Small pericardial and pleural effusions }\end{array}$ & $\begin{array}{l}\text {-Unexplained persistent } \\
\text { low-level cTn elevation } \\
\text {-Significant BNP or NT- } \\
\text { proBNP elevation } \\
\text {-BNP and troponin are } \\
\text { higher in AL. }\end{array}$ & $\begin{array}{l}\text {-Diffuse subendocardial LGE } \\
\quad \text {-Later transmural LGE } \\
\text {-Native myocardial T1 increased } \\
\text {-High extracellular volume }\end{array}$ \\
\hline
\end{tabular}

AL: light chain amyloidosis; AV: atrioventricular; BNP: brain natriuretic peptide; CTN: cardiac troponin; GLS: global longitudinal strain; LGE: late gadolinium enhancement; LV: left ventricular; NT-proBNP: N-terminal proBNP; RV: right ventricle

Adapted from Papinglotis G, Basmpana L, Farmakis D. Cardiac Amyloidosis: epidemiology, diagnosis and therapy. ESC. 2021; 19:19-21

indicated. The microscopic examination will show apple green birefringence with Congo red. ${ }^{5,8}$ (Figure 1)

In patients with heart failure due to amyloidosis, the maintenance of euvolemia will be essential, through fluid restriction and diuretics. There is no evidence for the use of beta blockers, neprilysin, angiotensin, or aldosterone receptor inhibitors . On the contrary, they can produce hypotension, exposing an autonomic neuropathy. ${ }^{5}$

The first and only medication indicated to reduce cardiovascular mortality and cardiovascular hospitalization in adults with hATTR or wtATTR is tafamidis, in a daily dose of $80 \mathrm{mg}$, which stabilizes the TTR tetramer and reduces the formation of TTR. ${ }^{8,9}$

Heart transplantation plays a small role due to the multiorgan nature of amyloidosis. Patients with AL have significant noncardiac amyloidosis and are not suitable candidates for heart transplantation, except when they have autologous stem cell transplantation or high-dose chemotherapy. Patients with wtATTR have the disease clinically isolated to the heart and would, thus, appear to be more suitable candidates. However, most patients are diagnosed in their seventh or eighth decade of life and are excluded based on their age. Patients with hATTR are younger and may be candidates if amyloid neuropathy is absent or mild.., 8

Other mechanisms of therapies continue to be investigated, such as the suppression of the TTR protein, through liver transplantation or genetic silencers, and the elimination of deposits by doxycycline and epigallocatechin-3-gallate. ${ }^{1,8}$

In summary, CA is an entity that is being diagnosed more frequently. The use of non-invasive techniques, such as scintigraphy with 99mTc-PYP, cardiac magnetic resonance, and light chains in blood and urine allow early identification and treatment of this disease, whose incidence and prevalence are still uncertain. 
Editorial

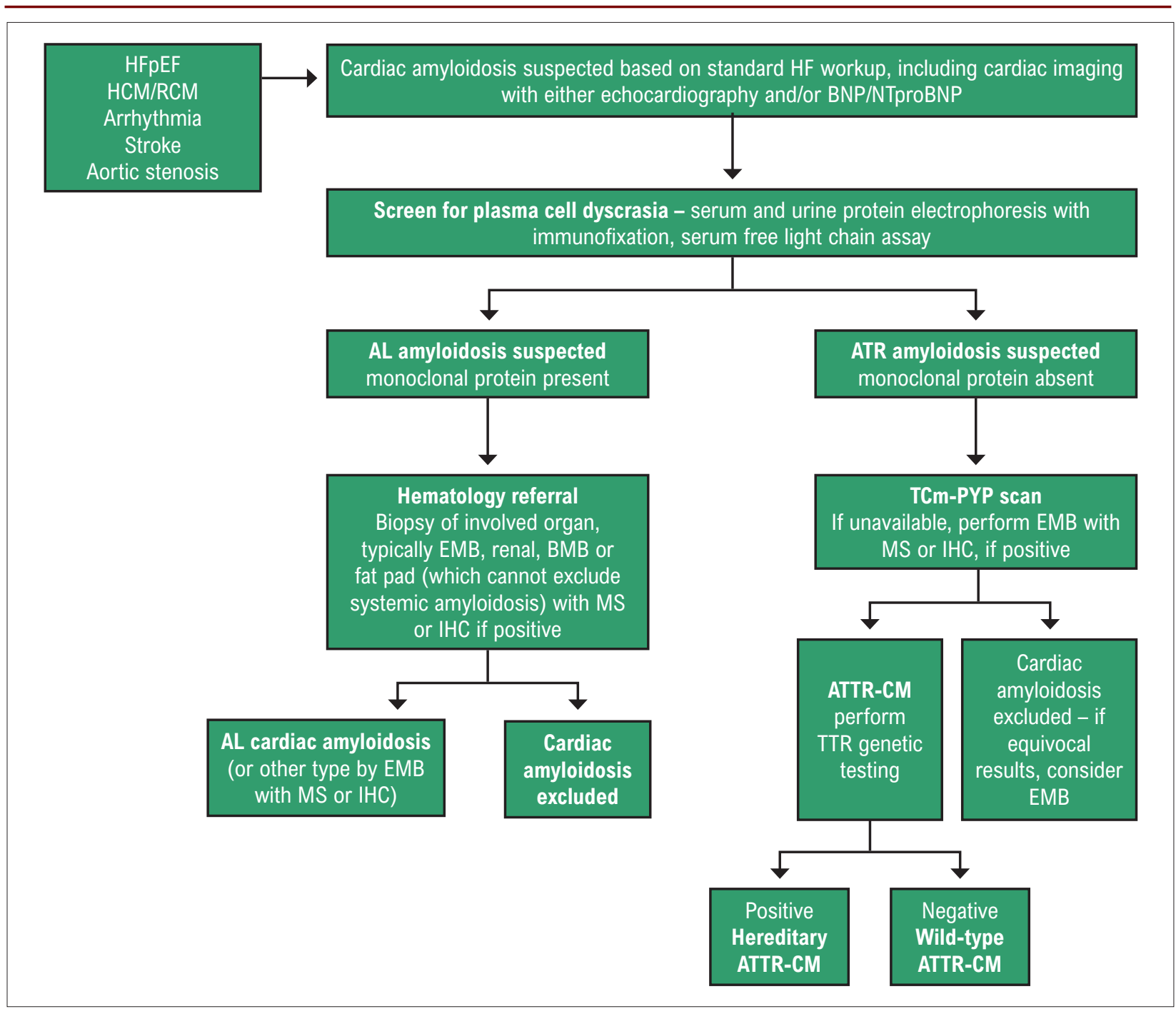

Figure 1-Diagnostic algorithm for evaluating suspected cardiac amyloidosis AL: Light chain amyloidosis; ATTR: transthyretin amyloidosis; ATTR-CM: transthyretin amyloid cardiomyopathy; BMB: bone marrow biopsy; BNP: b-type natriuretic peptide; CMR: cardiac magnetic resonance imaging; EMB: endomyocardial biopsy; HCM: hypertrophic cardiomyopathy; HF: heart failure; HFpEF: heart failure preserved ejection fraction; IHC: immunohistochemistry; MS: mass spectrometry; PYP: pyrophosphate; RCM: restrictive cardiomyopathy. Adapted from Fine M, Davis M, Anderson K, Delgado D, et al. Canadian Cardiovascular Society/Canadian Heart Failure Society Joint Position Statement on the Evaluation and Management of Patients with Cardiac Amyloidosis. CJCA. 2020; 20:322-334

\section{References}

1. García-Pavía P, Tomé-Esteban MT, Rapezzi C. Amiloidosis. También una Enfermedad del Corazón. Rev Esp Cardiol. 2011;64(9):797-808. doi: 10.1016/j.recesp.2011.05.003.

2. Rapezzi C, Lorenzini M, Longhi S, Milandri A, Gagliardi C, Bartolomei I, et al. Cardiac Amyloidosis: The Great Pretender. Heart Fail Rev. 2015;20(2):11724. doi: 10.1007/s10741-015-9480-0.

3. Fontana M, Ćorović A, Scully P, Moon JC. Myocardial Amyloidosis: The Exemplar Interstitial Disease. JACC Cardiovasc Imaging. 2019;12(11):2345-56. doi: 10.1016/j.jcmg.2019.06.023.

4. González-López E, Gallego-Delgado M, Guzzo-Merello G, Haro-Del Moral FJ, Cobo-Marcos M, Robles C, et al. Wild-Type Transthyretin Amyloidosis as a Cause of Heart Failure with Preserved Ejection Fraction. Eur Heart J. 2015;36(38):2585-94. doi: 10.1093/eurheartj/ehv338.

5. Gertz MA, Benson MD, Dyck PJ, Grogan M, Coelho T, Cruz M, et al. J Am Coll Cardiol. 2015;66(21):2451-66. doi: 10.1016/j.jacc.2015.09.075.
6. Falk RH, Alexander KM, Liao R, Dorbala S. AL (Light-Chain) Cardiac Amyloidosis: A Review of Diagnosis and Therapy. J Am Coll Cardiol. 2016;68(12):1323-41. doi: 10.1016/j.jacc.2016.06.053.

7. SipeJD, Benson MD, Buxbaum JN, Ikeda SI, Merlini G, Saraiva MJ, etal. Amyloid Fibril Proteins and Amyloidosis: Chemical Identification and Clinical Classification International Society of Amyloidosis 2016 Nomenclature Guidelines. Amyloid. 2016;23(4):209-13. doi: 10.1080/13506129.2016.1257986.

8. Fine NM, Davis MK, Anderson K, Delgado DH, Giraldeau G, Kitchlu A, et al. Canadian Cardiovascular Society/Canadian Heart Failure Society Joint Position Statement on the Evaluation and Management of Patients with Cardiac Amyloidosis. Can J Cardiol. 2020;36(3):322-34. doi: 10.1016/j. cjca.2019.12.034.

9. Maurer MS, Schwartz JH, Gundapaneni B, Elliott PM, Merlini G, WaddingtonCruz M, et al. Tafamidis Treatment for Patients with Transthyretin Amyloid Cardiomyopathy. N Engl J Med. 2018;379(11):1007-16. doi: 10.1056/ NEJMoa1805689. 


\section{Editorial}

